\title{
Automatic detection, consistent mapping, and training
}

\author{
SIU L. CHOW \\ The University of Wollongong, Wollongong, New South Wales, Australia
}

\author{
(W. J. Lovegrove, Sponsor)
}

\begin{abstract}
Results from two experiments showed that a flat display-size function was found under the consistent mapping (CM) condition despite the facts that there was no extensive CM training and that the stimulus-response (S-R) consistency was only an intrasession manipulation. A confounding factor might be responsible for the fact that the consistent and the varied S-R mapping conditions gave rise to different display-size functions in Schneider and Shiffrin's (1977) study. Their claim that automatic detection and controlled search are qualitatively different is also discussed.
\end{abstract}

When a multi-element stimulus is processed one element at a time, a controlled search is said to be taking place because the central processor does not have the required capacity to process multiple elements simultaneously. An automatic mode of processing takes place when the central processor can handle several elements simultaneously. The important question is what gives rise to a controlled or an automatic process.

Schneider and Shiffrin's (1977; Shiffrin \& Schneider, 1977) answer to the question is a two-process model that can be summarized by the following four assertions: (1) Controlled search can be set up readily, whereas extensive training involving a consistent stimulus-response (S-R) mapping is necessary for forming an automatic process, called "automatic detection." (2) Controlled search and automatic detection are qualitatively different activities. (3) The automatic-detection process is mediated by an automatic response, the activation of a node in the long-term store. (4) An automatic response may interfere with a controlled search process if a previously consistent S-R mapping is violated.

In their consistent mapping (CM) condition, a subject was trained to detect a letter among digits (or a digit among letters). In their varied mapping (VM) condition, a subject was trained to detect a letter among other letters (or a digit among other digits). Data collected after extensive training gave rise to flat and nonflat displaysize functions for the CM and VM conditions, respectively.

However, there might be a confounding between the physical appearance and the categorical membership of the stimuli used. This confounding was found by Schneider and Shiffrin (1977) only under the CM condition. Furthermore, they used $40-, 80$, and $120-\mathrm{msec}$

This research was supported by a Category A research grant from the University of Wollongong to the author. Requests for reprints should be sent to Siu L. Chow, Department of Psychology, The University of Wollongong, P.O. Box 1144, Wollongong, New South Wales, Australia 2500 . stimulus durations under the CM condition, but 120-, 200-, 400-, 600-, and 800-msec durations under the VM condition. The detection displays were subjected to both forward and backward masking. Moreover, those locations not filled by an alphanumeric item when the display size was smaller than four were filled by the mask stimulus. The subjects might be able to distinguish between a mask and an alphanumeric item only when the stimulus durations were long (i.e., in the VM condition). Consequently, the subjects might have effectively treated every display as though the display size was four in the CM condition. This might account for the flat display-size function under the $\mathrm{CM}$ condition and the nonflat function under the VM condition. The probable contributions of these procedural features were examined in this report.

\section{GENERAL METHOD}

\section{Subjects}

Two separate groups of 12 subjects were recruited from the first-year psychology students at the University of Wollongong for the two experiments. They were naive as to the purpose of the experiment. Their participation earned them five bonus marks toward their final grades.

\begin{abstract}
Apparatus
Stimulus displays were prepared and presented with a PDP/11-23 microcomputer driving a CIT-100 CRT. The type of phosphor used in the CRT is P-31 (green), which decays to the minimum discernible brightness in a well lit room in $38 \mu \mathrm{sec}$, a condition used in this study. The stimulus duration was timed with a programmable KWV-11A clock under the control of the program.
\end{abstract}

\section{Materials}

Three sets of alphanumeric items were used. The first set consisted of all numerals, except 1 and 0 . Two sets of consonants were selected on the basis of their shapes. The angular set consisted of $F, H, K, M$, $\mathrm{N}, \mathrm{W}, \mathrm{X}$, and $\mathrm{Z}$. The roundish set consisted of $\mathrm{B}, \mathrm{C}, \mathrm{D}, \mathrm{G}, \mathrm{P}, \mathrm{Q}, \mathrm{R}$, and $S$. Two or fewer of these three sets were used in any one experimental session.

The detection display was made up of the four corners of an imaginary square placed in the center of the CRT. All four corners were occupied when the display size was four. If the detection display size was one or two, the unoccupied locations were either left blank (the fillerabsent condition) or filled with the \# sign (the filler-present condition). 
Each alphanumeric item was made up of a $7 \times 9$ (points) grid subtending a visual angle of $20 \mathrm{~min}$ horizontally and $28 \mathrm{~min}$ vertically. A mask display always preceded the detection display. The mask display was made up of four dot patterns in the four corners of the imaginary square. The mask was a stimulus made up of all 63 points of the $7 \times 9$ grid. This display served to minimize any uncertainty regarding the locations of the items in the detection display.

\section{Design}

A $2 \times 2 \times 2 \times 3$ factorial design with repeated measures on all factors was used in both experiments. The two levels of the first factor were the absence or presence of backward masking. The second factor was the absence or presence of filler items when the detection display size was smaller than 4 (i.e., the filler-absent and filler-present conditions). The third factor was the type of S-R mapping, namely, consistent mapping (CM) or varied mapping (VM). The three levels of the last factor, detection display size, were 1,2 , and 4 . The S-R mapping was a betweensession manipulation (see Table 1). For every 1 of the 12 treatment combinations within a session, the target appeared six times in each of the four corners of the imaginary square.

\section{Procedure}

Subjects were tested individually in five $1 / 2$-h sessions, the first of which was a practice session. Data from the practice session (Session 1) were not included in the analysis. The order of testing for an individual subject was determined by the four rows of the Latin square shown under Sessions 2 through 4 in Table 1.

There were 300 trials in a session. As the S-R mapping factor was a between-session manipulation, there were effectively only 12 treatment combinations within a session (i.e., absence or presence of backward masking; absence or presence of filler items; and 1,2, or 4 items in the detection display). Each of these 12 treatment combinations was tested 24 times. Consequently, only 288 of the 300 trials were experimental trials. A target was present in half of the 24 trials tested under a particular treatment combination; no target was present in the other half Data from the first 12 warm-up trials in a session were not analyzed. The 288 experimental trials were randomly presented. The 12 warm-up trials were randomly chosen from the 12 treatment combinations.

The sequence of events in a trial was as follows. A $500-\mathrm{msec}, 1000-\mathrm{Hz}$ tone signaled the onset of a new trial. Half a second after the offset of the warning tone, a fixation point (viz., the + sign) was presented for $500 \mathrm{msec}$. Next, the target item was presented for $500 \mathrm{msec}$. After a 500-msec blank period, four masks occupying the corners of an imaginary square appeared for $500 \mathrm{msec}$. The detection display followed immediately for $20 \mathrm{msec}$. When applicable, four masks reappeared and stayed on until the subject responded. The CRT was left blank when the backward-mask present was not applicable. Subjects were instructed to respond as accurately as possible.

\section{EXPERIMENT 1}

The main objective of Experiment 1 was to re-examine whether the joint occurrence of CM mapping and extensive training was necessary for automatic detection when the target and nontarget items were readily distinguishable in shape. The theoretical expectation of the twoprocess model is that, with inexperienced subjects, both the CM and VM conditions should give rise to a linear display-size function with a negative slope significantly different from zero when detection accuracy is being measured. The subsidiary objective was to examine whether the presence of filler items in Schneider and Shiffrin's (1977) Experiments 1 and 2 could have differential effects in the CM and VM conditions. Numerals and angular letters were used in Experiment 1 (see Table 1).
Table 1

Order of Testing Adopted in the Two Experiments

\begin{tabular}{lccccc}
\hline & \multicolumn{5}{c}{ Session } \\
\cline { 2 - 6 } Subjects* & 1 & 2 & 3 & 4 & 5 \\
\hline $1,5,9$ & 11 & 11 & 12 & 21 & 22 \\
$2,6,10$ & 11 & 12 & 21 & 22 & 11 \\
$3,7,11$ & 11 & 21 & 22 & 11 & 12 \\
$4,8,12$ & 11 & 22 & 11 & 12 & 21 \\
\hline
\end{tabular}

11 = Consistent mapping with a numeral as a target in Experiment 1 and a roundish letter as a target in Experiment 2;12 = Consistent mapping with a letter as a target in Experiment 1 and an angular letter as a target in Experiment 2;21 = Varied mapping with a numeral as a target in Experiment 1 and a roundish letter as a target in Experiment 2; 22 = Varied mapping with a letter as a target in Experiment 1 and an angular letter as a target in Experiment 2. *Different groups of 12 subjects participated in Experiments 1 and 2.

Table 2

Linear Regression Analyses on the Display-Size Functions of Experiments 1 and 2

\begin{tabular}{lccccc}
\hline & \multicolumn{2}{c}{ Experiment 1 } & & \multicolumn{2}{c}{ Experiment 2 } \\
\cline { 2 - 3 } \cline { 5 - 6 } & $\mathrm{CM}$ & $\mathrm{VM}$ & & $\mathrm{CM}$ & $\mathrm{VM}$ \\
\hline Intercept & 0.532 & 0.544 & & 0.542 & 0.529 \\
Slope & -0.010 & $-0.047 *$ & & -0.016 & $-0.045 \dagger$ \\
\hline
\end{tabular}

$\mathrm{CM}=$ consistent mapping, $\mathrm{VM}=$ varied mapping. ${ }^{*}(2)=-2.336$, $p<.05 .+t(2)=-2.440, p<.05$.

\section{Results}

The dependent variable was the proportion of correct responses (i.e., hits and correct rejections), corrected for guessing (called genuine proportion correct) with the following equation:

$$
\mathrm{P}_{\mathrm{r}}=2 \times \mathrm{P}_{\mathrm{o}}-1 \text {, }
$$

where $\mathrm{P}_{\mathrm{o}}$ is the obtained proportion correct, and $\mathrm{P}_{\mathrm{r}}$ is the genuine proportion correct (i.e., correct guesses excluded).

The genuine proportion correct was subjected to a $2 \times 2 \times 2 \times 3$ ANOVA with repeated measures on all factors. All four main effects were significant at the .05 level [for S-R mapping, $F(1,11)=4.7$; for filler (absence or presence), $F(1,11)=14.8$; for backward masking, $F(1,11)=80.52$; for display size, $F(2,22)=13.88]$. Also significant at the .05 level was the S-R mapping $\times$ display size interaction $[F(2,22)=6.18]$. None of the other interactions was significant.

An examination of the S-R mapping $\times$ display size interaction revealed that only the linear $x$ linear trend was significant $[F(1,11)=12.07, p<.05]$. Consequently, a linear regression analysis (on display size) was conducted separately for the CM and the VM conditions. As may be seen from the left-hand panel of Table 2, only the slope of the display-size function under the VM condition was significant $[t(2)=-2.336, p<.05]$.

\section{Discussion}

The absence of a filler $\times$ S-R mapping $\times$ display size interaction suggests that the different display-size functions obtained by Schneider 
and Shiffrin (1977) under the CM and the VM conditions could not be due to the presence of filler items. The subjects' CM performance in this experiment was expected to mimic their VM performance because the subjects were not trained as extensively as were their counterparts in Schneider and Shiffrin's (1977) study. However, the CM condition gave a flat function, whereas the VM condition gave a nonflat function. This observation is contrary to the two-process model.

\section{EXPERIMENT 2}

The existence of an automatic response in Shiffrin and Schneider's (1977) model needs to be considered with reference to the suggestions that (1) all items in a multiitem array are fully analyzed, (2) selection is made at the response-selection stage (J. A. Deutsch \& D. Deutsch, 1963; Shiffrin \& Geisler, 1973), (3) nodes in the longterm store that have been consistently mapped to a response over an extended period of time activate themselves in the presence of the eliciting stimuli, (4) the nodes in question may be some previously established higher order nodes (such as the node for the category of numerals) or they may be established experimentally (Shiffrin \& Schneider, 1977, Experiment 3), and (5) these nodes are more sophisticated than physical features, such as angles and straight lines.

Angular and roundish letters were used in Experiment 2 (see Table 1). Consequently, subjects under the CM condition in this experiment could not appeal to any previously established higher order long-term store node because there was not any categorical distinction between the target and nontarget sets. The theoretical expectation of the two-process model is that the CM and the VM conditions should give rise to identical display-size accuracy functions with a negative slope significantly smaller than zero.

\section{Results}

Subjects' genuine proportion correct data were subjected to a $2 \times 2 \times 2 \times 3$ ANOVA with repeated measures on all factors. The ANOVA revealed that all main effects were significant at the .05 level [for S-R mapping, $F(1,11)$ $=18.92$; for filler (absence or presence), $F(1,11)=6.23$; for backward mask, $F(1,11)=85.58$; for display size, $F(2,22)=13.76]$. Also significant were (1) the S-R mapping $\times$ display size interaction $[F(2,22)=6.76$; the linear trend component is significant, $F(1,11)=5.71$, $p<.05]$, and (2) the filler $\times$ display size interaction $[F(2,22)=3.7$; the linear trend is also significant, $F(1,11)$ $=8.21$ ]. No other interaction was significant. As in Experiment 1 , only the display-size function under the VM condition gave a significant slope $[t(2)=-2.440$, $p<.05]$ (see Table 2).

\section{Discussion}

Apart from the addition of the significant filler $\times$ display size interaction, the data from this experiment replicated those of Experiment 1. The significant S-R mapping $\times$ display size interaction is contrary to the theoretical expectation of the two-process model, because (1) there was no extensive training and (2) there was no preexperimental categorical distinction between target and nontarget items.

\section{GENERAL DISCUSSION}

The purpose of this study is not to dispute Schneider and Shiffrin's (1977) and Shiffrin and Schneider's (1977) empirical findings. Nor does it question the notion of automaticity as such. Consequently, this study is not incompatible with the type of automaticity envisaged by Posner and Snyder (1975a, 1975b). Rather, it is an attempt to highlight the fact that Schneider and Shiffrin's (1977) conclusions about a type of automaticity, based on information other than physical features, are not warranted by their data.

In terms of the two-process model, two procedural features in the present study should have worked against a flat display-size function under the CM condition. First, subjects in these experiments were insufficiently trained on the consistent S-R mapping by Schneider and Shiffrin's (1977) procedural standard (viz., over $10 \mathrm{~h}$ of training). It is possible that $300 \mathrm{CM}$ training trials were insufficient to establish an automatic response based on some higher order information, other than physical features, because Schneider and Shiffrin (1977) have not specified the minimal amount of training required for establishing automaticity. This feature of the model renders it so flexible that it can account for virtually any pattern of data. A theory that cannot be disproved is not a satisfactory one.

Second, as can be seen from Table 1, the consistent mapping manipulation in this study was not actually consistent after all. Although the S-R mapping might be consistent within a session, it was not consistent across sessions for any individual (see Table 1). In view of Shiffrin and Schneider's (1977) and Schneider, Dumais, and Shiffrin's (1984) contention, reversing the target and nontarget sets across sessions should have prevented the subjects from achieving automatization. However, the contrary is true in the present two experiments. The inescapable conclusion is that something other than extensive CM training is responsible for the flat display-size function in the CM condition and a nonflat function in the VM condition in Schneider and Shiffrin's (1977) study. The likely candidate is the preattentive selection in terms of physical features.

The present findings have an implication on the claim that $\mathrm{CM}$ and VM training necessarily give rise to qualitatively different processes. The subjects in Schneider and Shiffrin's (1977) Experiment 3 were presented with a multi-item array over several frames in which none, one, or two targets might be present. The subjects had to indicate how many targets were present (as opposed to merely indicating that a target was present).

In Schneider and Shiffrin's (1977) multiframe paradigm, the multiple targets (when applicable) might be in the same frame or 1,2, or 4 frames apart (a variable called spacing). In their Experiment 3a, Schneider and Shiffrin (1977) found that the CM and the VM conditions gave rise to different spacing functions (see Figure 18 of Schneider \& Shiffrin, 1977). This became the basis of their claim that automatic and controlled processing were qualitatively different. In all of their subsequent arguments, the two spacing functions in their Figure 18 were used as indices of automatic and of controlled processing. This practice is unacceptable for the following reasons.

In their Experiment 3a, Schneider and Shiffrin (1977) used a memory size of 2 and a display size of 2 only. In their Experiment 3b, they used a memory size of 2 and a display size of 4 for the $C M$ condition. For the VM condition, they used (1) a memory size of 2 together with a display size of 4 , and (2) a memory size of 4 together with a display size of 2 . In other words, neither the memory nor the perceptual load was varied in Experiments $3 a$ and $3 b$. Yet detection or search performance was said to be a function of the product of the memory size times the display size (Experiments 1 and 2, Schneider \& Shiffrin, 1977). In the absence of any load manipulation, it is not reasonable to claim that the $\mathrm{CM}$ and the VM conditions necessarily give rise to automatic detection and controlled search, respectively (see also Cheng, 1985).

Schneider and Shiffrin (1977) appealed to the fact that their subjects had been extensively trained under the two S-R mapping conditions. This argument is acceptable only if it can be assumed that their extensive CM training is the necessary and sufficient condition for automaticity in the sense they envisaged. However, this is the very assumption being questioned here. That is, in view of the present findings, the spacing functions in Schneider and Shiffrin's (1977) Figure 18 are not neces- 
sarily indicative of a qualitative difference between automatic detection and controlled search.

In their response to Cheng (1985), Schneider and Shiffrin (1985) appealed to the observation that their subjects' performance at two different levels of memory set size was comparable after extensive practice and a transfer to the CM condition (Shiffrin \& Schneider, 1977, Experiment 3). However, the reason why the absence of a memory set size effect in this context necessarily indicates automaticity is not made clear.

From their third experiment on, Schneider and Shiffrin (1977; Shiffrin \& Schneider, 1977; as well as Schneider and his associates in subsequent papers, e.g., Fisk \& Schneider, 1983) equated CM (as an experimental manipulation) with automatic processing (as a hypothetical mechanism). That it is improper to equate an experimental procedure with a theoretical notion has been cogently shown by Baddeley (1976), who distinguished between short-term memory (an experimental procedure) and short-term store (a theoretical notion). Likewise, the fact that CM is used does not necessarily mean that automatic processing is involved. Yet, it has been asserted, "The processing occurring in CM paradigms is referred to as automatic processing" (Fisk \& Schneider, 1983, p. 178; their emphasis).

In summary, it can be concluded that Schneider and Shiffrin (1977) had not unambiguously excluded preattentive selection in terms of physical features as the basis of the flat CM display-size function. Consequently, their conclusion regarding an automatic response based on information more sophisticated than physical features is not warranted. This report also contributes to the recent exchange between Ryan (1983) and Shiffrin and Schneider (1984) as well as to that between Cheng (1985) and Schneider and Shiffrin (1985) regarding the validity and usefulness of the two-process model.

\section{REFERENCES}

BADDELEy, A. D. (1976). The psychology of memory. New York: Basic Books.

Cheng, P. W. (1985). Restructuring versus automaticity: Alternative accounts of skill acquisition. Psychological Review, 92, 414-423.
Deutsch, J. A., \& Deutsch, D. (1963). Attention: Some theoretical considerations. Psychological Review, 70, 80-90.

FISK, A. D., \& SCHNEIDER, W. (1983). Category and word search: Generalizing search principles to complex processing. Journal of $E x$ perimental Psychology: Learning, Memory, \& Cognition, 9, 177-195.

POSNER, M. I., \& SNYDER, R. R. (1975a). Attention and cognitive control. In R. L. Solso (Ed.), Information processing and cognition: The Loyola Symposium (pp. 55-85). Hillsdale, NJ: Erlbaum.

PosNer, M. I., \& SNYDER, R. R. (1975b). Facilitation and inhibition in the processing of signals. In P. M. A. Rabbitt \& S. Dornic (Eds.), Attention and performance $V$ (pp. 669-682). New York: Academic Press.

RYAN, C. (1983). Reassessing the automaticity-control distinction: Item recognition as a paradigm case. Psychological Review, 90, 171-178.

SChNeIDER, W., Dumais, S. T., \& ShIFfrin, R. M. (1984). Automatic and control processing and attention. In R. Parasuraman \& D. R. Davies (Eds.), Varieties of attention (pp. 1-27). Orlando, FL: Academic Press.

SCHNEIDER, W., \& SHIFFrIN, R. M. (1977). Controlled and automatic human information processing: I. Detection, search, and attention. Psychological Bulletin, 84, 1-66.

SCHNEIDER, W., \& SHIFFrin, R. M. (1985). Categorization (restructuring) and automatization: Two separable factors. Psychological Review, 92, 424-428.

Shiffrin, R. M., \& GeISLER, W. S. (1973). Visual recognition in a theory of information processing. In R. L. Solso (Ed.), Contemporary Issues in Cognitive Psychology: The Loyola Symposium (pp. 53-101). Washington, DC: Winston.

SHIFFrin, R. M., \& SCHNEIDER, W. (1977). Controlled and automatic human information processing: II. Perceptual learning, automatic attending, and a general theory. Psychological Review, 84, 127-190.

SHIFFrin, R. M., \& SCHNEIDER, W. (1984). Automatic and controlled processing revisited. Psychological Review, 91, 269-276.

(Manuscript received for publication June 30, 1986.) 